\title{
Methods and difficulties of different ways in gaining central line access: a literature review
}

\author{
Muhammad A. Alkholy ${ }^{1 *}$, Jumanah T. Albarakati², Mustafa M. Rawas ${ }^{3}$, Lujain O. Rayes ${ }^{4}$, \\ Manal A. Alghamdi ${ }^{5}$, Belal Naaman', Wejdan A. Alyamani ${ }^{6}$, Awadh M. Alqahtani ${ }^{7}$, \\ Sultan S. Alrashidi ${ }^{8}$, Amin A. Amin', Talal S. Alyahyawi ${ }^{7}$
}

\author{
${ }^{1}$ Department of Emergency Medicine, ${ }^{3}$ Department of Anesthesia, East Jeddah Hospital, Jeddah, Saudi Arabia \\ ${ }^{2}$ Department of Intensive Care Unit, King Abdulaziz Specialist Hospital, Taif, Saudi Arabia \\ ${ }^{4}$ Department of Emergency Medicine, Batterjee Medical College, Jeddah, Saudi Arabia \\ ${ }^{5}$ College of Medicine, Alfarabi Colleges, Riyadh, Saudi Arabia \\ ${ }^{6}$ Internship, Batterjee Medical College, Jeddah, Saudi Arabia \\ ${ }^{7}$ Department of Emergency, Aseer Central Hospital, Abha, Saudi Arabia \\ ${ }^{8}$ Department of Emergency, Madina Munwara General Hospital, Medina, Saudi Arabia \\ ${ }^{9}$ Department of Emergency, Ministry of Health, Medina, Saudi Arabia
}

Received: 15 December 2020

Accepted: 31 December 2020

\author{
*Correspondence: \\ Dr. Muhammad A. Alkholy, \\ E-mail: masaied@moh.gov.sa
}

Copyright: (c) the author(s), publisher and licensee Medip Academy. This is an open-access article distributed under the terms of the Creative Commons Attribution Non-Commercial License, which permits unrestricted non-commercial use, distribution, and reproduction in any medium, provided the original work is properly cited.

\begin{abstract}
The central line is one of the vital techniques in medicine and it is widely used for critically ill patients' resuscitation. Proper knowledge of the proper techniques and understanding how to choose the perfect central vein for each patient is considered a vital skill for physicians. In this study, we reviewed the literature to provide a simple guide for physicians. PubMed, Medline, Web of Science, Embase, Google Scholar, and Scopus using the following search terms ("Central line" or "central catheter") and ("method" or "procedure" or "complication"). We included any study that reports the methods and difficulties of different ways of gaining central line access. Based on our results, the proper choice of the central line and the site of insertion are the determining factors for the success of the procedure and decreasing the risk of complications. The ultrasound-guided approach is considered the best option to avoid any injury of the vital structures.
\end{abstract}

Keywords: Central line, Central catheter, Ultrasound-guided approach

\section{INTRODUCTION}

The central line is considered a crucial and vital technique for the resuscitations of critically ill patients. ${ }^{1}$ It is usually used for fluid resuscitation, delivers multiple medication and infusion chemotherapy. ${ }^{1}$ One of the drugs that are usually given in the central line is vesicant drugs that usually harmful to the skin and muscle tissues if given through another route. ${ }^{2}$ The usual site of the central line is the subclavian vein, the internal jugular vein, and the femoral vein. ${ }^{1,3}$ The most common site is the subclavian vein as it is associated with less risk of infection and thrombosis. ${ }^{4}$ However, the choice of the site depends on the condition of the patients along with complications of each type. ${ }^{4}$ Despite being a life-saving technique in critically ill patients, if not done carefully and correctly, life-threatening complications usually occur. Arrhythmia and cardiac arrest can occur in case of incorrect insertion of the central line. That is why an ultrasound-guided approach is recommended for any central vein. ${ }^{5,6}$ 
The most common complication is an infection of the central line which can be avoided by simple procedures as proper training of the staff, use of central line coated with antibacterial, flushing of central line with saline, and heparin. ${ }^{5}$ Other non-infectious complications include arterial and venous injury which occurs in case of faulty techniques. Hematoma, hemorrhage, and thrombosis are also reported to be common. ${ }^{5-8}$ Notwithstanding, all these complications can be avoided by a simple understanding of the technique of insertion of the central line, proper identification of the veins, and proper choice of the necessary and suitable type of the central line. ${ }^{4}$ Thus, in this review, we reviewed the literature regarding the types of central lines, indications, and complications associated with the central line.

\section{SEARCH STRATEGY}

A systematic search was conducted to identify relevant studies in the following databases: PubMed, Medline, Web of Science, Embase, Google Scholar, and Scopus. The following search terms were used ("central line" or "central catheter") and ("method" or "procedure" or "complication"). The reference lists were manually searched to identify additional relevant studies meeting inclusion criteria. We included any study that reports the methods and difficulties of different ways of gaining central line access. No restrictions were applied.

\section{TYPES OF CENTRAL VEIN CATHETERS}

Many types of central vein catheters are present in the clinical field and the choice of optimum type is usually dependent on the nature of the treatment given and the plan of treatment whether it is for a long time or not. There are five types of catheters: non-tunneled catheters, tunneled catheters, implanted ports, dialysis catheters, and peripherally inserted central catheters. ${ }^{9}$

The non-tunneled catheters are considered the most simple and easiest catheters to be inserted and that is why it is used for emergency and acute situations. ${ }^{9}$ The disadvantage of this type is that they cannot be kept for a long time and only used for a short time to serve its purpose. It was reported that it was associated with a high rate of infections and thrombosis if kept for a long time. It is estimated that it can be kept for two weeks to one month. ${ }^{9}$

Tunneled catheters overcome the disadvantage associated with the insertion of the non-tunneled catheters as it can be used for over one month. The rate of infection is lower than other types of catheters making it the perfect fit for the long duration use and when intravenous access is needed multiple times. ${ }^{9}$

Implanted ports are usually used as it has fewer scars and better cosmetic appearance. It was reported that they had less infection incidence compared to other types. However, it requires a surgical procedure that takes more time and requires an advanced level of skills compared to other types making it unfit for emergencies. ${ }^{9}$

Another well-known catheter is dialysis catheters which are usually indicated for haemodialysis and continuous renal replacement techniques. The continuous renal replacement techniques are different from haemodialysis that it is usually associated with slower fluid dialysis than haemodialysis providing better control, thus, hemodynamic stability. ${ }^{10}$ Moreover, it is used in medical emergencies like septic shock or sepsis and acute kidney injury. For haemodialysis catheters, it is usually indicated in cases of metabolic acidosis, hypokalaemia, and high serum creatinine $(>10 \mathrm{mg} / \mathrm{dl}) .{ }^{11}$ The dialysis catheters are characterized by wide pores; thus, the patients require heparin intake to avoid clotting of these pores and dysfunction of the catheters. The dialysis catheters can be tunneled or non-tunneled. The tunneled type is usually used for long-duration treatment while the non-tunneled are usually used in case of emergencies. ${ }^{10}$

Peripherally inserted central catheters provide central access through upper arm veins and then the catheters are guided into superior vena cava on the right side of the heart. ${ }^{9,11}$ The catheters are usually thin and flexible tubes and can be inserted for a long duration of up to months. It is usually required when the peripheral venous access integrity is required. ${ }^{9,11}$

\section{DISCUSSION}

\section{Sites of central line placements and methods of insertion}

The most common sites of insertion of central line catheters are the subclavian vein, the internal jugular vein, and the femoral vein. To gain access to these veins, ultrasound is usually used to guide the insertion of the catheters into these veins. ${ }^{9}$

The subclavian vein is a common site for the central line; it originates from the axillary vein which at the lateral border of the first rib, it becomes the subclavian vein then it continues below the clavicle where it goes towards the sternum till it crosses the medial border of the anterior scalene muscles. ${ }^{12}$ The insertion of the central line into this vein usually at the point where the vein crosses the clavicle as it is done by an infraclavicular approach. ${ }^{13}$ The doctor usually stands on the ipsilateral side of the desired vein then turns the head of the patients to the opposite side to gain clear visualization of the anatomic landmarks. At the junction between the medial and middle one-third of the clavicle, the needle is pointed towards the sternal notch. The point of needle entry is usually $1-2 \mathrm{~cm}$ inferior and lateral to this junction. ${ }^{12,13}$

Another site of the central line insertion is the internal jugular vein. ${ }^{9}$ The course of the internal jugular vein is more complex as it comes near the vital structure in the neck making any mistake in the insertion of the needle increase the risk of injury of these structures. ${ }^{14,15}$ During its 
course, it lies anterior to the Vagus nerve and anterior lateral to the internal and common carotid arteries. The vein arises from the sigmoid sinus in the posterior cranial fossa then passes through the jugular foramen in the skull to the neck and thorax where it joins the subclavian vein to form the brachiocephalic vein. ${ }^{9}$ The right internal jugular vein drains into the superior vena cava while the left does not end immediately into the superior vena cava. There are two ways of insertion of the central line in the jugular vein wither through a centralized approach where the needle is pointed to the ipsilateral nipple and the needle enters the apex of the triangle. However, this approach is obsolete now due to ultrasound-guided insertion. ${ }^{14,15}$ The right jugular vein is more common to use as it is away from the apex of the lung unlike the left jugular vein which has a higher risk of lung perforation. ${ }^{14,15}$

The femoral vein is considered the last choice for the central line as it is associated with many complications during insertion. The femoral vein is formed by the joining of the superficial femoral vein and deep femoral vein. ${ }^{1}$ Once it reaches the inguinal ligament, it continues as an external iliac vein then the external iliac vein combine with the internal iliac vein to form a common iliac vein that will end eventually in the inferior vena cava draining into the right atrium. ${ }^{2}$ The insertion of a femoral vein catheter is easy as the doctor palpates the femoral artery then the femoral vein will be medial to it and in case of difficulty, the ultrasound-guided insertion is used. ${ }^{2}$

\section{The complications associated with a central line insertion}

The complication of central line insertion can be either immediate or delayed complications. The immediate complications occur after the insertion of the central line. ${ }^{8}$ It can be categorized into cardiac, vascular, pulmonary, and catheter insertion procedures (Figure 1). ${ }^{6}$

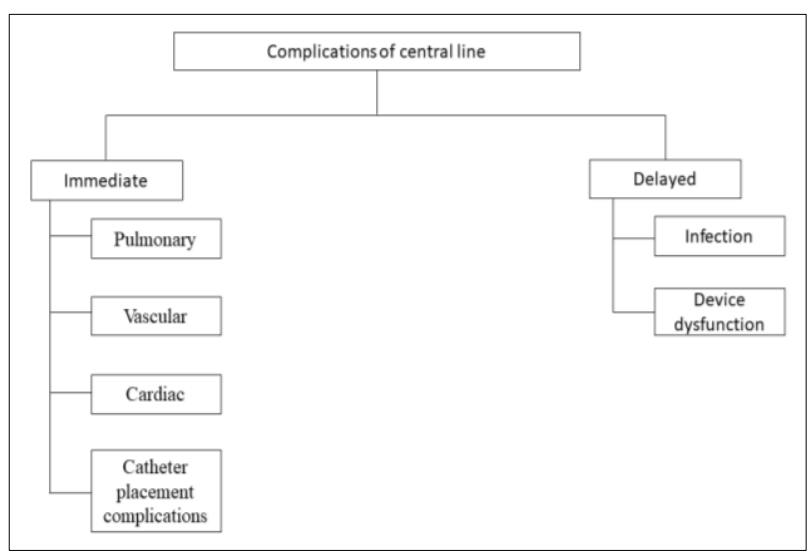

Figure 1: Summary of central line complications.

Cardiac complications are one of the most prominent immediate complications after central line insertion. Arrhythmias and insertion of the guidewire into the right atrium resulting in premature atrial and ventricular arrhythmias. ${ }^{7,16}$ Usually, it is solved by the guide-wire depth and monitoring for the early diagnosis of arrhythmias. Once diagnosed, advanced cardiac life support should be started. Cardiac arrest can happen in the case of supraventricular tachycardia.,16 Other complications include right ventricular complications caused by faulty insertion of the central line into the pulmonary artery leading to cardiac tamponade. It is usually treated by pericardiocentesis. ${ }^{7,16}$

Vascular complications usually include arterial, venous injury, bleeding, and hematoma formation. ${ }^{4,6-8,16}$ Arterial injuries usually occur with the femoral vein central line. Arterial puncture usually occurs in $4.2 \%$ to $9.3 \%$ of cases. ${ }^{15,17}$ The incidence of the arterial puncture is the same with or without an ultrasound-guided approach. ${ }^{13,18}$ The most difficult decision in arterial puncture whether they should leave the catheter in its place or remove it as both had a high risk of stroke, thrombosis, and haemorrhage, pseudoaneurysm, and AV fistula. ${ }^{15,17}$ For venous injury, there were reported lacerations in the mediastinal vessels and right atrium. It has been caused by the insertion of a dilator or catheter that is pushed against the vessel wall. The repair of these lesions usually requires surgical repair resulting in thrombosis of the blood vessels. ${ }^{15,17}$

Another well documented is hematoma formation in $4.7 \%$ of all cases. The hematoma is usually benign and resolves spontaneously and did not require any repair. The complicated hematoma usually located deep in the mediastinum and requires surgical drainage. Infection of the hematoma can lead to abscess formation. ${ }^{8}$

Pulmonary complications usually arise from the central line in the internal jugular vein resulting in chylothorax and chylopericardium. ${ }^{19}$ Recurrent laryngeal nerve injury was reported in $1.6 \%$ of central line insertions as it occurs due to accidental trauma or hematoma formation around the nerve. ${ }^{5,14,16,20}$ Other injured nerves include the sympathetic chain, the phrenic nerves, and brachial plexus.,14,16 Air embolism is also one of the documented complications in some patients but usually, it is small and insignificant. ${ }^{21}$

For delayed complications, infections of the central line led to sepsis and shock..$^{4-6,8}$ The incidence is usually at least 80 cases per 100000 patients per year. ${ }^{22}$ The reported mortality rate was 12 to $25 \%$. It usually results from the biofilm formation resulting from Staphylococcus aureus and Staphylococcus epidermidis. Broad-spectrum antibiotics are usually used for treatment. ${ }^{22,23}$

Device dysfunction occurs when one of the components of the central catheter is impaired resulting in the formation of the fibrin sheath, catheter fracture, thrombosis, and obstruction. Fibrin sheath is the most commonly encountered problem resulting in tube obstruction. It is usually treated using fibrinolytics. ${ }^{1,20}$

Central line fracture is another dysfunction in the case of a subclavian vein if it is inserted for a long time. It can lead to life-threatening conditions like endocarditis, cardiac 
perforation, and arrhythmias. ${ }^{5-7}$ It is caused by the pinchoff syndrome. It results from compression of the catheters by the first rib and the clavicle. Venous stenosis has a prevalence of $41 \%$ and results from long-term catheterization and it can be treated using a venous stent. ${ }^{5-}$

\section{CONCLUSION}

The central line is considered an essential skill that physicians should know how to do appropriately as it is lifesaving as well as life-threatening if incorrectly inserted. More care and the use of an ultrasound-guided approach is necessary to avoid any complications. Also, the proper choice of the type of catheter will greatly decrease the complications.

\section{Funding: No funding sources \\ Conflict of interest: None declared \\ Ethical approval: Not required}

\section{REFERENCES}

1. Bhutta ST, Culp WC. Evaluation and management of central venous access complications. Tech Vasc Interv Radiol. 2011;14(4):217-24.

2. Taylor RW, Palagiri AV. Central venous catheterization. Crit Care Med. 2007;35(5):1390-6.

3. Vascular Access Work Group. Clinical practice guidelines for vascular access. Am J Kidney Dis. 2006;48(1):176-247.

4. McGee DC, Gould MK. Preventing complications of central venous catheterization. New Engl J Med. 2003;348(12):1123-33.

5. Kornbau C, Lee KC, Hughes GD, Firstenberg MS. Central line complications. Int $\mathbf{J}$ Crit illness Injury Sci. 2015;5(3):170-8.

6. Kusminsky RE. Complications of central venous catheterization. J Am Coll Surgeons. 2007;204(4):681-96.

7. Bowdle A. Vascular complications of central venous catheter placement: evidence-based methods for prevention and treatment. J Cardiothoracic Vascular Anesthesia. 2014;28(2):358-68.

8. Vats HS. Complications of catheters: tunneled and nontunneled. Adv Chronic Kidney Dis. 2012;19(3):188-94.

9. Bannon MP, Heller SF, Rivera M. Anatomic considerations for central venous cannulation. Risk Management Healthcare Policy. 2011;4:27-39.

10. Claure-Del Granado R, Mehta RL. Fluid overload in the ICU: evaluation and management. BMC Nephrol. 2016;17(1):109-11.

11. Hanafusa N. Application of Continuous Renal Replacement Therapy: What Should We Consider Based on Existing Evidence? Blood Purification. 2015;40(4):312-9.
12. Wang HE, Sweeney TA. Subclavian central venous catheterization complicated by guidewire looping and entrapment. J Emerg Med. 1999;17(4):721-4.

13. Fragou M, Gravvanis A, Dimitriou V, Papalois A, Kouraklis G, Karabinis A, et al. Real-time ultrasound-guided subclavian vein cannulation versus the landmark method in critical care patients: a prospective randomized study. Crit Care Med. 2011;39(7):1607-12.

14. Konichezky S, Saguib S, Soroker D. Tracheal puncture. A complication of percutaneous internal jugular vein cannulation. Anaesthesia. 1983;38(6):572-4.

15. Shah PM, Babu SC, Goyal A, Mateo RB, Madden RE. Arterial misplacement of large-caliber cannulas during jugular vein catheterization: case for surgical management. J Am Coll Surgeons. 2004;198(6):93944.

16. Bossert T, Gummert JF, Bittner HB, Barten M, Walther T, Falk V, et al. Swan-Ganz catheter-induced severe complications in cardiac surgery: right ventricular perforation, knotting, and rupture of a pulmonary artery. J Cardiac Surg. 2006;21(3):292-5.

17. Guilbert M-C, Elkouri S, Bracco D, Corriveau MM, Beaudoin N, Dubois MJ, et al. Arterial trauma during central venous catheter insertion: Case series, review and proposed algorithm. J Vasc Surg. 2008;48(4):918-25.

18. Powell JT, Mink JT, Nomura JT, Levine BJ, Jasani $\mathrm{N}$, Nichols WL, et al. Ultrasound-guidance can reduce adverse events during femoral central venous cannulation. J Emerg Med. 2014;46(4):519-24.

19. Teichgraber UKM, Nibbe L, Gebauer B, Wagner HJ. Inadvertent puncture of the thoracic duct during attempted central venous catheter placement. Cardiovasc Intervent Radiol. 2003;26(6):569-71.

20. Khouzam RN, Soufi MK, Weatherly M. Heparin infusion through a central line misplaced in the carotid artery leading to hemorrhagic stroke. J Emerg Med. 2013;45(3):87-9.

21. Hsu M, Trerotola SO. Air embolism during insertion and replacement of tunneled dialysis catheters: a retrospective investigation of the effect of aerostatic sheaths and over-the-wire exchange. J Vasc Intervent Radiol. 2015;26(3):366-71.

22. Vital signs: central line-associated blood stream infections--United States, 2001, 2008, and 2009. Morbidity Mortality Weekly Rep. 2011;60(8):243-8.

23. Pronovost P, Needham D, Berenholtz S, Sinopoli D, $\mathrm{Chu} \mathrm{H}$, Cosgrove $\mathrm{S}$, et al. An intervention to decrease catheter-related bloodstream infections in the ICU. New Engl J Med. 2006;355(26):2725-32.

Cite this article as: Alkholy MA, Albarakati JT, Rawas MM, Rayes LO, Alghamdi MA, Naaman B, et al. Methods and difficulties of different ways in gaining central line access: a literature review. Int $\mathbf{J}$ Community Med Public Health 2021;8:881-4. 